\title{
Saturation with chiral interactions and consequences for finite nuclei
}

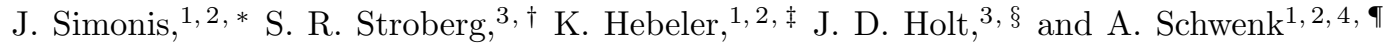 \\ ${ }^{1}$ Institut für Kernphysik, Technische Universität Darmstadt, 64289 Darmstadt, Germany \\ ${ }^{2}$ ExtreMe Matter Institute EMMI, GSI Helmholtzzentrum für Schwerionenforschung GmbH, 64291 Darmstadt, Germany \\ ${ }^{3}$ TRIUMF, 4004 Wesbrook Mall, Vancouver, BC V6T 2A3, Canada \\ ${ }^{4}$ Max-Planck-Institut für Kernphysik, Saupfercheckweg 1, 69117 Heidelberg, Germany
}

\begin{abstract}
We explore the impact of nuclear matter saturation on the properties and systematics of finite nuclei across the nuclear chart. Using the ab initio in-medium similarity renormalization group (IM-SRG), we study ground-state energies and charge radii of closed-shell nuclei from ${ }^{4} \mathrm{He}$ to ${ }^{78} \mathrm{Ni}$, based on a set of low-resolution two- and three-nucleon interactions that predict realistic saturation properties. We first investigate in detail the convergence properties of these Hamiltonians with respect to model-space truncations for both two- and three-body interactions. We find one particular interaction that reproduces well the ground-state energies of all closed-shell nuclei studied. As expected from their saturation points relative to this interaction, the other Hamiltonians underbind nuclei, but lead to a remarkably similar systematics of ground-state energies. Extending our calculations to complete isotopic chains in the $s d$ and $p f$ shells with the valence-space IM-SRG, the same interaction reproduces not only experimental ground states but two-neutron-separation energies and first excited $2^{+}$states. We also calculate radii with the valence-space IM-SRG for the first time. Since this particular interaction saturates at too high density, charge radii are still too small compared with experiment. Except for this underprediction, the radii systematics is, however, well reproduced. Our results highlight the importance of nuclear matter as a theoretical benchmark for the development of next-generation chiral interactions.
\end{abstract}

\section{INTRODUCTION}

A central goal of nuclear theory is an accurate ab initio description of nuclei from the valley of stability to the limits of existence based on a common Hamiltonian including theoretical uncertainties. Recent progress in chiral effective field theory (EFT) [1, 2] and renormalization group methods [3, 4] methods provide a framework for addressing this goal. The enhanced convergence properties of the resulting interactions and methodological advances in many-body theory, e.g., with coupledcluster (CC) theory 5, self-consistent Green's function methods [6], or the in-medium similarity renormalization group (IM-SRG) 7], which exhibit a polynomial scaling in the mass number, have increased the reach of ab initio calculations to the medium-mass region or beyond. While these large-space methods are still limited to closed-shell and neighboring nuclei, or to even-even systems, doubly open-shell nuclei can be accessed by ab initio valence-space methods [8 12].

These developments have enabled a clear demonstration of the importance of three-nucleon $(3 \mathrm{~N})$ forces for understanding the structure of medium-mass nuclei 13, 14 and for realistic saturation properties of nuclear matter [15-19. Indeed, the role of $3 \mathrm{~N}$ forces in saturation was suggested long ago [20, 21], but difficulties in formulating consistent $3 \mathrm{~N}$ forces and solving the

\footnotetext{
* E-mail: simonis@theorie.ikp.physik.tu-darmstadt.de

$\dagger$ E-mail: sstroberg@triumf.ca

¥ E-mail: kai.hebeler@physik.tu-darmstadt.de

$\S$ E-mail: jholt@triumf.ca

ฯ E-mail: schwenk@physik.tu-darmstadt.de
}

resulting many-body problem hindered progress. More recently, the difficulty has been in constructing a chiral interaction which simultaneously reproduces saturation, few-body observables, and spectroscopy. In Ref. 22, a good description of ground-state energies in the region of ${ }^{16} \mathrm{O}$ was obtained using a chiral two- and three-nucleon interaction. However, this interaction yields radii which are too small 23, and increasingly severe overbinding for heavier nuclei [24]. An alternative approach [25], fitting directly to some medium-mass nuclei successfully reproduced saturation, but two-nucleon (NN) scattering data were only fit up to $35 \mathrm{MeV}$.

In this work, we investigate ground-state energies and charge radii of finite nuclei based on chiral low-resolution $\mathrm{NN}$ and $3 \mathrm{~N}$ interactions with realistic saturation properties, which also reproduce scattering data with high precision. We use the closed-shell and valence-space formulation of the IM-SRG. This enables a unique and broad access across the nuclear chart. In Sec. IA we describe the Hamiltonians used in this work, while Sec. IB gives a short overview on the IM-SRG formalism for closed-shell nuclei and the decoupling of valence-space interactions for open-shell nuclei. The model-space convergence of ground-state energies and charge radii for different resolution scales and low-energy couplings is studied in detail in Sec. III Finally, Sec. III presents valence-space results for different $s d$ - and $p f$-shell isotopic chains, including first results for radii in the valence-space IM-SRG.

\section{A. Chiral interactions and saturation}

At the NN level, we start from the next-to-next-tonext-to-leading order $\left(\mathrm{N}^{3} \mathrm{LO}\right) 500 \mathrm{MeV}$ potential of En- 
tem and Machleidt (EM) [26. We then use the similarity renormalization group (SRG) 3, 27] to evolve this interaction to a series of low-resolution scales $\lambda_{\mathrm{NN}}=$ $1.8,2.0,2.2 \mathrm{fm}^{-1}$. Taking chiral EFT as a general lowmomentum basis and assuming the long-range couplings $c_{i}$ to be invariant under the SRG transformation, we combine each SRG-evolved NN interaction with the leading $\mathrm{N}^{2} \mathrm{LO} 3 \mathrm{~N}$ forces [28, 29, where the $c_{i}$ couplings in the two-pion-exchange $3 \mathrm{~N}$ interaction are taken consistently with the NN interaction: $c_{1}=-0.81 \mathrm{GeV}^{-1}, c_{3}=$ $-3.2 \mathrm{GeV}^{-1}, c_{4}=5.4 \mathrm{GeV}^{-1}$. In addition, to probe uncertainties in the $c_{i}$ couplings, we use $3 \mathrm{~N}$ forces with the $c_{i}$ values obtained from the Nijmegen $\mathrm{NN}$ partial-wave analysis (PWA): $c_{1}=-0.76 \mathrm{GeV}^{-1}, c_{3}=-4.78 \mathrm{GeV}^{-1}$, $c_{4}=3.96 \mathrm{GeV}^{-1}$ [30] for the $\lambda_{\mathrm{NN}}=2.0 \mathrm{fm}^{-1}$ interaction. For all Hamiltonians, the low-energy couplings $c_{D}$, $c_{E}$ in the $3 \mathrm{~N}$ one-pion-exchange and $3 \mathrm{~N}$ contact interaction have been fit to the ${ }^{3} \mathrm{H}$ binding energy and ${ }^{4} \mathrm{He}$ charge radius using Faddeev and Faddeev-Yakubovsky calculations [16] with a nonlocal $3 \mathrm{~N}$ regulator and cutoff $\Lambda_{3 \mathrm{~N}}=2.0 \mathrm{fm}^{-1}$.

These chiral NN+3N Hamiltonians were first employed to study symmetric [16] and, more recently, also asymmetric nuclear matter [31, 32]. The first application to finite nuclei was in a valence-space study of $s d$-shell isotopes [33] and in coupled-cluster calculations of selected $\mathrm{Ca}$ [34, 35] and $\mathrm{Ni}$ isotopes [36. Of particular importance to this work is that in symmetric nuclear matter the $\lambda_{\mathrm{NN}} / \Lambda_{3 \mathrm{~N}}=1.8 / 2.0(\mathrm{EM})$ interaction yields an energy per particle in good agreement with the empirical value (at saturation density with a Hartree-Fock spectrum slighty too bound [32]), although at a somewhat too high density. The other interactions 2.0/2.0 (EM), 2.2/2.0 (EM), 2.0/2.0 (PWA) saturate at decreasingly smaller energy and density [32].

\section{B. In-medium SRG}

In the IM-SRG approach 7], starting from the full $\mathrm{NN}+3 \mathrm{~N}$ Hamiltonian, we first solve the Hartree-Fock equations to obtain a suitable reference state. We then rewrite the Hamiltonian in normal-ordered form:

$$
\begin{aligned}
H= & E_{0}+\sum_{i j} f_{i j}\left\{a_{i}^{\dagger} a_{j}\right\}+\frac{1}{4} \sum_{i j k l} \Gamma_{i j k l}\left\{a_{i}^{\dagger} a_{j}^{\dagger} a_{l} a_{k}\right\} \\
& +\frac{1}{36} \sum_{i j k l m n} W_{i j k l m n}\left\{a_{i}^{\dagger} a_{j}^{\dagger} a_{k}^{\dagger} a_{n} a_{m} a_{l}\right\},
\end{aligned}
$$

where the braces denote a string of creation and annihilation operators normal ordered with respect to the reference state, and the resulting in-medium zero-, one-, and two-body operators, $E_{0}, f$, and $\Gamma$, respectively, represent the starting values for the IM-SRG flow equations. In the normal-ordered two-body approximation, in which we work, the residual three-body term $W$ is discarded. We use the Magnus formulation presented in Ref. 37] to generate an explicit unitary transformation that decouples the reference state from excitations. This transformation can subsequently be applied to any operator, in particular the radius operator discussed below. For calculations of open-shell nuclei, we use the valence-space formulation of the IM-SRG (VS-IM-SRG) [8, 11, 38, based on the ensemble normal-ordering discussed in Ref. [12, which captures the bulk effects of residual $3 \mathrm{~N}$ forces among valence nucleons. A valence-space Hamiltonian is then produced specifically for each nucleus, which is diagonalized with the NuShellX shell-model code [39] to obtain ground- and excited-state energies in the valence space.

\section{CLOSED-SHELL NUCLEI}

In this section, we analyze the model-space convergence of closed-shell nuclei based on the four chiral low-resolution $\mathrm{NN}+3 \mathrm{~N}$ interactions introduced in Sec. IA. In the calculations we employ an angularmomentum-coupled basis built from single-particle spherical harmonic-oscilator (HO) states with quantum numbers $e=2 n+l \leqslant e_{\text {Max }}$. We employ partial-wave decomposed $3 \mathrm{~N}$ matrix elements in a Jacobi-momentum basis and include partial waves up to the total three-body angular momentum $\mathcal{J} \leqslant 9 / 2$. Furthermore in order to manage computational storage requirements, we introduce an additional cut $e_{1}+e_{2}+e_{3} \leqslant E_{3 \text { Max }}<3 e_{\text {Max }}$ for $3 \mathrm{~N}$ matrix elements. For the analysis of the convergence behavior presented below, we study $e_{\mathrm{Max}} / E_{3 \mathrm{Max}}=$ 10/14, 12/14, 14/14, 14/16, and 14/18.

In addition to ground-state energies, we also investigate the convergence behavior of charge radii. These results are obtained by normal-ordering and evolving the intrinsic proton mean-square radius operator (see, e.g., Ref. [41),

$$
R_{p}^{2}=\frac{1}{Z} \sum_{i}^{Z}\left(\vec{r}_{i}-\vec{R}\right)^{2}
$$

where $i$ runs over the proton coordinates, and $\vec{R}$ is the center-of-mass coordinate. Note that the proton meansquare radius operator is not free-space SRG evolved, consistent with the determination of the $3 \mathrm{~N}$ couplings to the charge radius of ${ }^{4} \mathrm{He}$. We obtain charge radii by applying the corrections arising from the mean-square charge radii of the proton and the neutron as well as the relativistic Darwin-Foldy and spin-orbit corrections:

$$
R_{\mathrm{ch}}=\sqrt{R_{p}^{2}+\left\langle r_{p}^{2}\right\rangle+\frac{N}{Z}\left\langle r_{n}^{2}\right\rangle+\frac{3}{4 M_{p}^{2} c^{4}}+\left\langle r^{2}\right\rangle_{\mathrm{so}}},
$$

with values of $\left\langle r_{p}^{2}\right\rangle$ and $\left\langle r_{n}^{2}\right\rangle$ taken from Ref. 42. The spin-orbit correction [4], is calculated from

$$
\left\langle r^{2}\right\rangle_{\mathrm{so}}=\frac{1}{Z} \sum_{i=1}^{A}\left\langle r_{i}^{2}\right\rangle_{\mathrm{so}}=-\frac{1}{Z} \sum_{i} \frac{\mu_{i}}{M^{2}}\left(\kappa_{i}+1\right),
$$



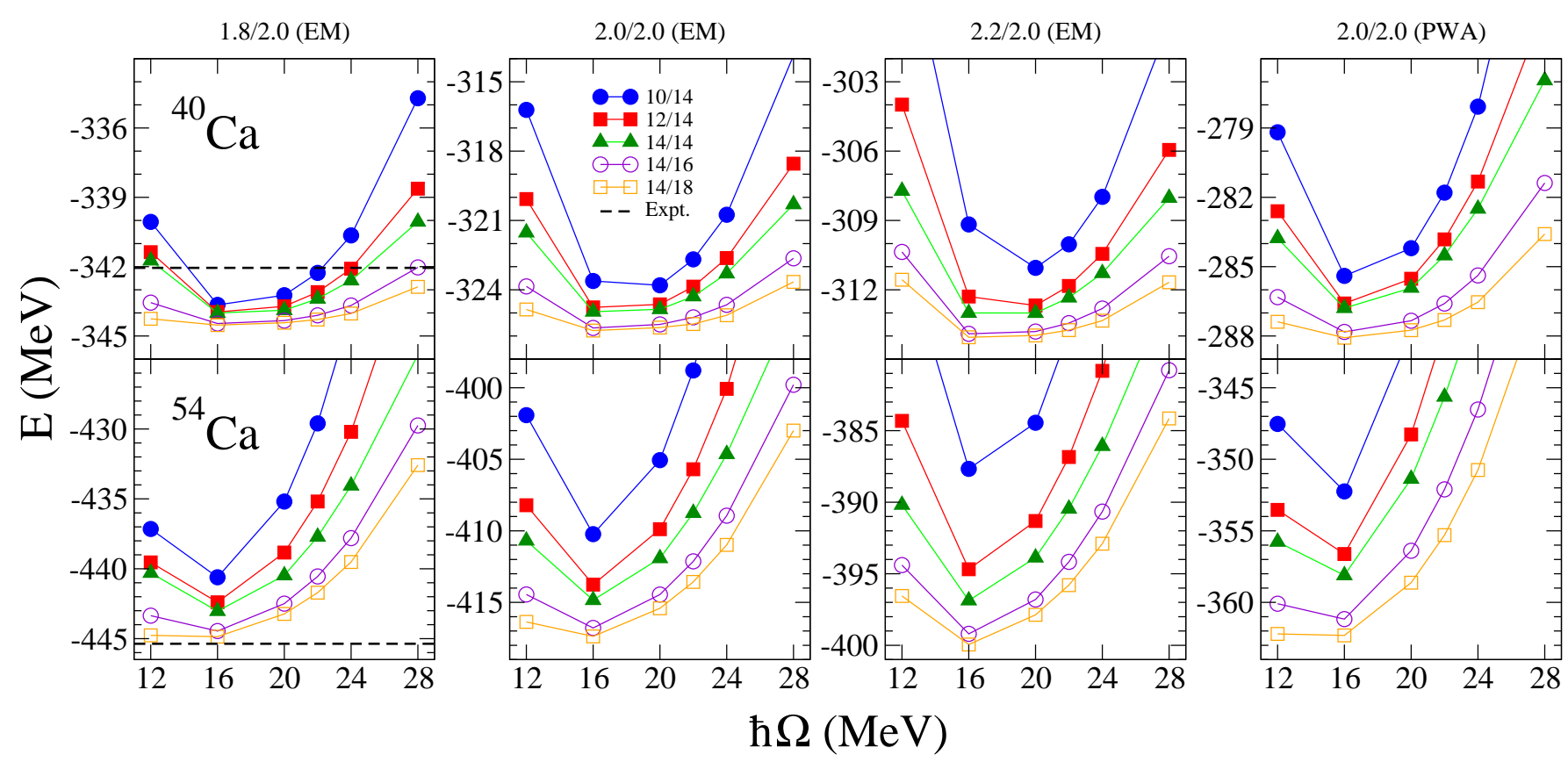

FIG. 1. Convergence of ground-state energies for ${ }^{40} \mathrm{Ca}$ (top panels) and ${ }^{54} \mathrm{Ca}$ (bottom panels) calculated with the closed-shell IM-SRG. The column heading specifies the input Hamiltonian: $\lambda_{\mathrm{NN}} / \Lambda_{3 \mathrm{~N}}=1.8 / 2.0(\mathrm{EM})(\mathrm{left}), 2.0 / 2.0$ (EM) (middle-left), 2.2/2.0 (EM) (middle-right), and 2.0/2.0 (PWA) (right). In each panel, we show results obtained for harmonic-oscillator frequencies $\hbar \Omega=(12-28) \mathrm{MeV}$ and different truncations of the single-particle basis $e_{\mathrm{Max}} / E_{3 \mathrm{Max}}=10 / 14$ (filled circles), $12 / 14$ (filled squares), 14/14 (filled triangles), 14/16 (empty circles), and 14/18 (empty squares). The experimental ground-state energies from the atomic mass evaluation (AME) 2012 [40, are given by the dashed lines.

\begin{tabular}{lcccc|cccc}
\hline \hline & \multicolumn{4}{c|}{} & \multicolumn{4}{c}{${ }^{78} \mathrm{Ni}$} \\
Hamiltonian & $(10 \rightarrow 12) / 14$ & $(12 \rightarrow 14) / 14$ & $14 /(14 \rightarrow 16)$ & $14 /(16 \rightarrow 18)$ & $(10 \rightarrow 12) / 14$ & $(12 \rightarrow 14) / 14$ & $14 /(14 \rightarrow 16)$ & $14 /(16 \rightarrow 18)$ \\
\hline $1.8 / 2.0(\mathrm{EM})$ & 1.8 & 0.6 & 1.4 & 0.4 & 3.3 & 0.9 & 4.4 & 2.0 \\
$2.0 / 2.0(\mathrm{EM})$ & 3.5 & 1.1 & 1.9 & 0.6 & 7.4 & 2.1 & 5.7 & 2.8 \\
$2.2 / 2.0$ (EM) & 7.0 & 2.2 & 2.3 & 0.7 & 14.7 & 5.0 & 6.6 & 3.4 \\
$2.0 / 2.0$ (PWA) & 4.4 & 1.5 & 3.1 & 1.1 & 9.2 & 3.0 & 9.6 & 5.0 \\
\hline \hline
\end{tabular}

TABLE I. Convergence of ground-state energies of ${ }^{54} \mathrm{Ca}$ and ${ }^{78} \mathrm{Ni}$ for the four Hamiltonians considered. The table lists the change in the ground-state energy when increasing $e_{\mathrm{Max}} \rightarrow e_{\mathrm{Max}}+2\left(E_{3 \mathrm{Max}} \rightarrow E_{3 \mathrm{Max}}+2\right)$ at fixed $E_{3 \mathrm{Max}}\left(e_{\mathrm{Max}}\right)$ for harmonicoscillator frequency $\hbar \Omega=16 \mathrm{MeV}$.

with the magnetic moments of the proton, $\mu_{p}=2.793 \mu_{N}$, and the neutron, $\mu_{n}=-1.913 \mu_{N}$, and the definition

$$
\kappa= \begin{cases}l, & j=l-\frac{1}{2} \\ -(l+1), & j=l+\frac{1}{2} .\end{cases}
$$

Additionally, two-body currents, due to the coupling of the photon to pions and to two nucleons, contribute to the charge radius, but this correction is neglected here.

In Fig. 1. we show the model-space convergence for ground-state energies of ${ }^{40} \mathrm{Ca}$ and ${ }^{54} \mathrm{Ca}$. The energy minima are almost independent of the four different $\mathrm{NN}+3 \mathrm{~N}$ interactions, typically located near $\hbar \Omega=16 \mathrm{MeV}$. While the ground-state energy of ${ }^{40} \mathrm{Ca}$ is well converged for the different Hamiltonians, in ${ }^{54} \mathrm{Ca}$ convergence from $e_{\operatorname{Max}} / E_{3 \operatorname{Max}}=10 / 14$ to $14 / 14$ is only obtained for the interactions with lower resolution scales $\lambda_{\mathrm{NN}}=1.8$ and $2.0 \mathrm{fm}^{-1}$. In Table II we list the change in the groundstate energy with increasing $e_{\text {Max }} / E_{3 \mathrm{Max}}$. We clearly see the 1.8/2.0 (EM) interaction is better converged from $e_{\operatorname{Max}} / E_{3 \mathrm{Max}}=12 / 14$ to $14 / 14$, where the energy decreases by only $0.6 \mathrm{MeV}$ total, compared with the $2.2 / 2.0$ (EM) interaction, where this decrease is $2.2 \mathrm{MeV}$.

In addition, we investigate the impact of increasing the $3 \mathrm{~N}$ cut $E_{3 \mathrm{Max}}=14 \rightarrow 18$ for $e_{\mathrm{Max}}=14$ both in Fig. 1 and Table I. In the case of the 1.8/2.0 (EM) interaction, the ground-state energy of ${ }^{54} \mathrm{Ca}$ decreases by $0.4 \mathrm{MeV}$ for $E_{3 \mathrm{Max}}=16 \rightarrow 18$, while this decrease of $0.7 \mathrm{MeV}$ is only slightly larger for the $2.2 / 2.0$ (EM) interaction, indicating both are relatively well converged in terms of $E_{3 \mathrm{Max}}$. The largest impact is seen with the 2.0/2.0 (PWA) interaction, where the difference amounts 

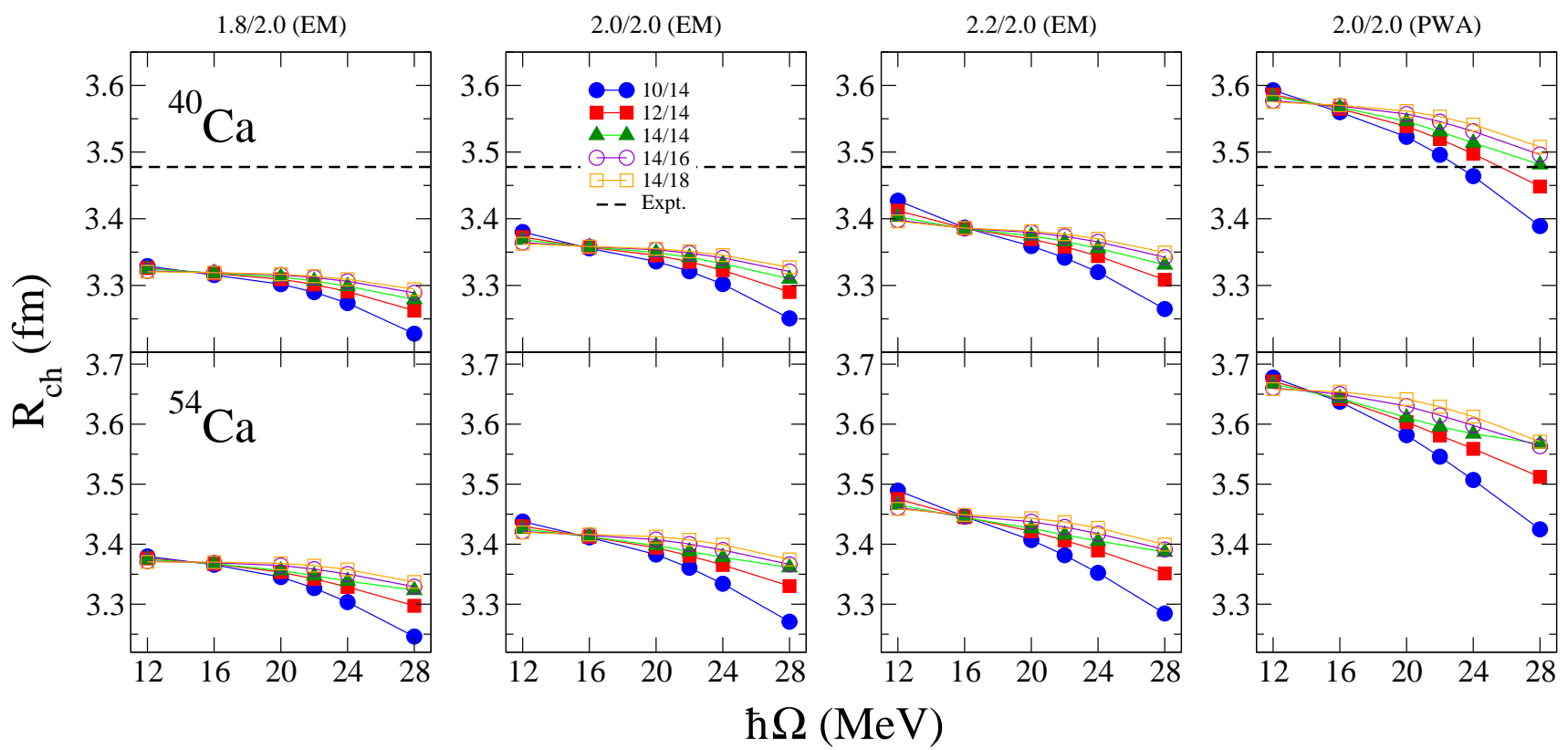

FIG. 2. Convergence of charge radii for ${ }^{40} \mathrm{Ca}$ (top panels) and ${ }^{54} \mathrm{Ca}$ (bottom panels) calculated with the closed-shell IM-SRG. The legend is as in Fig. 1. The experimental charge radius for ${ }^{40} \mathrm{Ca}$ [4] is given by the dashed line.

to $1.1 \mathrm{MeV}$. While the ground-state energies calculated with the 1.8/2.0 (EM) interaction agree with experiment to $\approx 1 \%$, the other three Hamiltonians predict energies that are significantly underbound.

In Fig. 2, we show the model-space convergence of the charge radii for ${ }^{40} \mathrm{Ca}$ and ${ }^{54} \mathrm{Ca}$. Although the groundstate energy of ${ }^{40} \mathrm{Ca}$ calculated from the 1.8/2.0 (EM) interaction is in remarkable agreement with experiment, the corresponding charge radius, shown in the left column of Fig. 2, is significantly smaller than experiment. With increasing SRG resolution scale $\lambda_{\mathrm{NN}}$, the charge radii increase but are still too small compared to experiment, while for the 2.0/2.0 (PWA) Hamiltonian, the calculated charge radius is instead somewhat too large. It will be very interesting to compare the charge-radii calculations for ${ }^{54} \mathrm{Ca}$, shown in the lower panels of Fig. 2 with future experimental results. Even the recent measurement of the charge radius of ${ }^{52} \mathrm{Ca} 35$, manifesting a strong increase from ${ }^{48} \mathrm{Ca}$ onward, could not be explained fully by ab initio coupled-cluster calculations.

In Fig. 3, we show the model-space convergence for ground-state energies of ${ }^{56} \mathrm{Ni}$ and ${ }^{78} \mathrm{Ni}$. Similar to the calcium isotopes (see Fig. 1) the minima in the ground-state energies are near $\hbar \Omega=16 \mathrm{MeV}$. The energies obtained from the 1.8/2.0 (EM) Hamiltonian are again in very good agreement with experiment, while the other three Hamiltonians give results that are underbound to different degrees. The increase in particle number from calcium to nickel clearly results in slower model-space convergence. As seen in Table I, enlarging the single-particle basis from $e_{\mathrm{Max}}=12 \rightarrow 14$ changes the ground-state energy of ${ }^{78} \mathrm{Ni}$ by $0.9 \mathrm{MeV}$ for the $1.8 / 2.0$ (EM) interaction at $\hbar \Omega=16 \mathrm{MeV}$, compared to $5.0 \mathrm{MeV}$ for the $2.2 / 2.0$
(EM) interaction. The change in energy when increasing the cut in the $3 \mathrm{~N}$ matrix elements from $E_{3 \mathrm{Max}}=16 \rightarrow 18$ is $2.0 \mathrm{MeV}$ for $1.8 / 2.0$ (EM), already not completely converged. This effect is even larger for 2.0/2.0 (EM) and 2.2/2.0 (EM) and maximal for 2.0/2.0 (PWA), where the change is $5.0 \mathrm{MeV}$. Again, for the 1.8/2.0 (EM) interaction, the agreement with experiment is good in both cases, but it is clear that the model space must be increased beyond $e_{\mathrm{Max}} / E_{3 \mathrm{Max}}=14 / 18$ to claim fully converged results in this region, and likely for any nucleus with $N, Z \gtrsim 50$. We also note the unusual behavior of the ${ }^{78} \mathrm{Ni}$ results for $e_{\mathrm{Max}} / E_{3 \mathrm{Max}}=14 / 14$ at $\hbar \Omega=28 \mathrm{MeV}$ in Fig. 3 is probably caused by truncation artifacts due to the $E_{3 \mathrm{Max}}$ cut.

In Fig. 4, we show the model-space convergence of the charge radii for ${ }^{56} \mathrm{Ni}$ and ${ }^{78} \mathrm{Ni}$. Similar to the calcium isotopes discussed above, we see a gradual increase with increasing SRG resolution scale and a larger value for the 2.0/2.0 (PWA) interaction. While the results for ${ }^{56} \mathrm{Ni}$ appear well converged for all starting Hamiltonians, this is less the case for ${ }^{78} \mathrm{Ni}$ either with respect to $e_{\text {Max }}$ or $E_{3 \text { Max }}$, and especially for the larger cutoffs and the 2.0/2.0 (PWA) interaction.

Finally, before studying the systematics of the groundstate energies and charge radii of closed shell nuclei, we compare our results to the coupled-cluster calculations of Hagen et al. 36] for the 1.8/2.0 (EM) interaction. Considering the same model-space truncation $e_{\mathrm{Max}} / E_{3 \mathrm{Max}}=14 / 16$ and harmonic-oscillator frequency $\hbar \Omega=16 \mathrm{MeV}$ we find good agreement within $\approx 1 \%$ for ${ }^{16} \mathrm{O}$ : $-127.2 \mathrm{MeV}$ (IM-SRG(2)) vs. $-128 \mathrm{MeV}$ $(\Lambda-\mathrm{CCSD}(\mathrm{T}))$; for ${ }^{40} \mathrm{Ca}:-344.5 \mathrm{MeV}$ vs. $-348 \mathrm{MeV}$; for ${ }^{48} \mathrm{Ca}: \quad-416.1 \mathrm{MeV}$ vs. $-419 \mathrm{MeV}$; and for ${ }^{78} \mathrm{Ni}$ : 

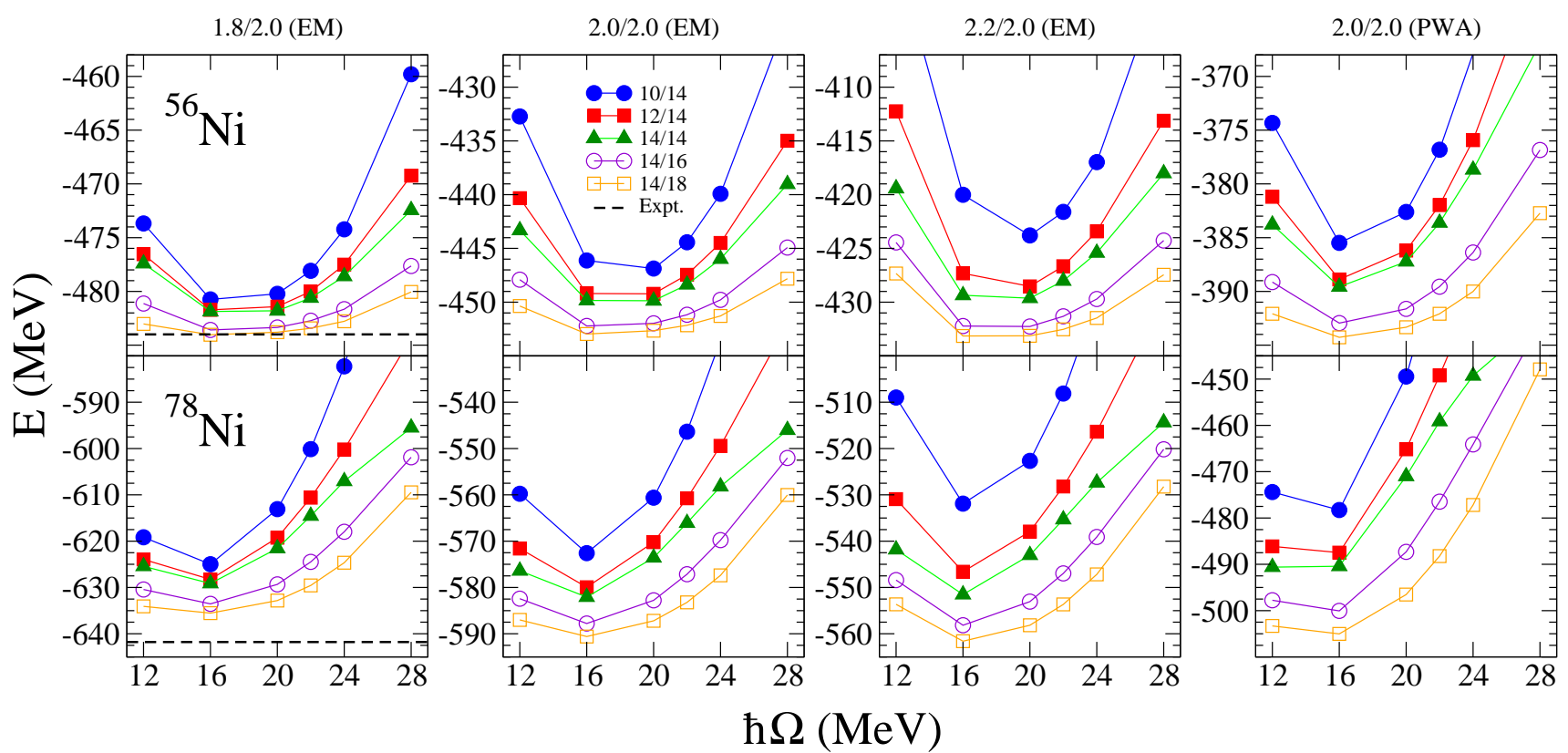

FIG. 3. Convergence of ground-state energies for ${ }^{56} \mathrm{Ni}$ (top panels) and ${ }^{78} \mathrm{Ni}$ (bottom panels) calculated with the closed-shell IM-SRG. The legend is as in Fig. 1] Note that the ground-state energy for ${ }^{78} \mathrm{Ni}$ is extrapolated.
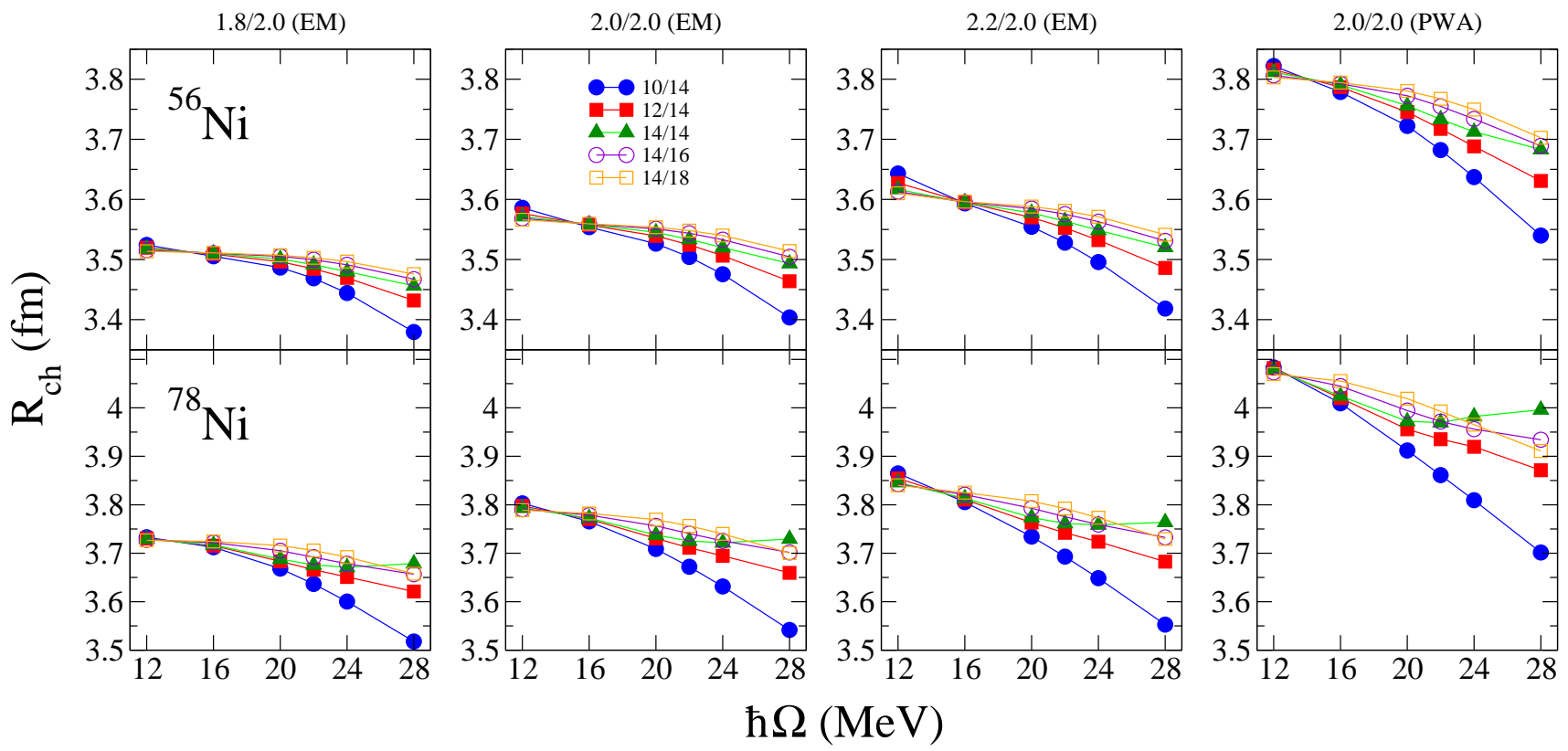

FIG. 4. Convergence of charge radii for ${ }^{56} \mathrm{Ni}$ (top panels) and ${ }^{78} \mathrm{Ni}$ (bottom panels) calculated with the closed-shell IM-SRG. The legend is as in Fig. 1 .

$-633.6 \mathrm{MeV}$ vs. $-637 \mathrm{MeV}$, while there is a difference of more than $3 \%$ for ${ }^{4} \mathrm{He}(-29.2 \mathrm{MeV}$ vs. $-28.2 \mathrm{MeV})$.

Finally, in Figs. 5 and 6 we show ground-state energies and charge radii, respectively, for selected closed-shell nuclei from ${ }^{4} \mathrm{He}$ to ${ }^{78} \mathrm{Ni}$. Except for the neutron-rich oxygen isotopes ${ }^{22,24} \mathrm{O}$ all calculated ground-state energies from the 1.8/2.0 (EM) interaction are in very good agreement with experiment. Interestingly the other three interac- tions follow the same pattern but are shifted by as much as $1.5 \mathrm{MeV} / \mathrm{A}$ in the case of the $2.0 / 2.0$ (PWA) interaction. The experimental charge radii are enclosed by the 2.2/2.0 (EM) and 2.0/2.0 (PWA) results, but the trend observed for the closed-shell nuclei studied in detail already above appears to hold at least up to ${ }^{78} \mathrm{Ni}$. That is, radii with $1.8-2.2 / 2.0$ are too small, but 2.0/2.0 (PWA) gives slightly too large radii. As in the case 


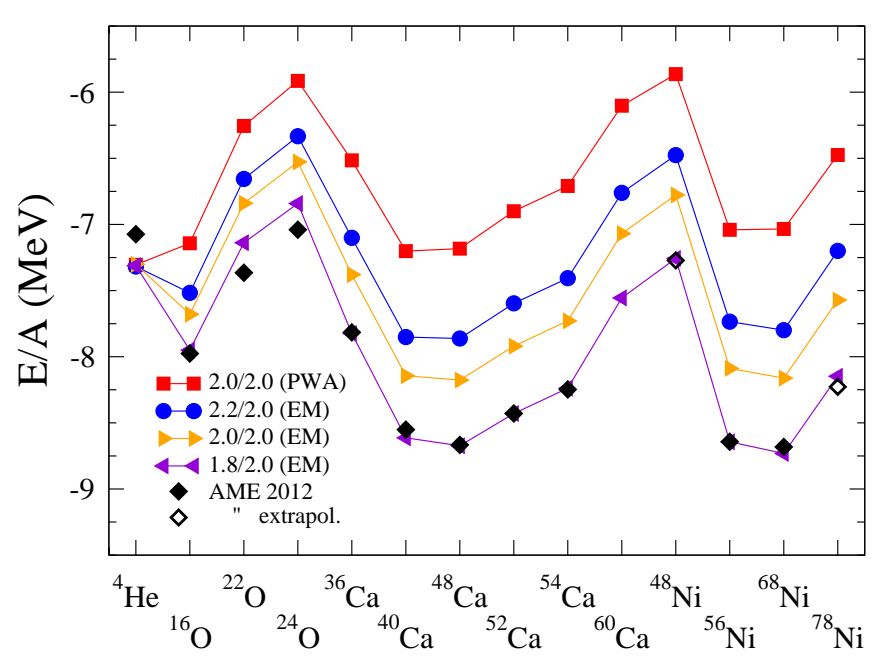

FIG. 5. Systematics of the energy per nucleon $E / A$ of closedshell nuclei from ${ }^{4} \mathrm{He}$ to ${ }^{78} \mathrm{Ni}$ calculated with the IM-SRG for the four Hamiltonians considered. The results are compared against experimental ground-state energies from the AME 2012 [40] (extrapolated for ${ }^{48,78} \mathrm{Ni}$ ).

of ground-state energies, the radii systematics is similar for all Hamiltonians; with mainly only a constant shift for the different interactions. This behavior for the ground-state energy and charge radii is reminiscent of the Coester-like line for the saturation points of the four Hamiltonians considered 32 .

\section{OPEN-SHELL ISOTOPIC CHAINS}

In this section, we move beyond closed-shell systems to explore ground- and excited-state systematics throughout a selection of isotopic chains in the $s d$ and $p f$ shells, namely sodium, sulfur, calcium, manganese, and nickel. The VS-IM-SRG method used here was shown to agree with large-space methods to better than $1 \%$ for groundstate energies [12].

We also calculate charge radii, less studied within the context of ab initio approaches [23, 25, 34, 35, 45], with the VS-IM-SRG for the first time, where the proton mean-square radius operator of Eq. (2) is transformed via the same unitary transformation as the Hamiltonian. This gives a valence-space radius operator to be used with valence-space wave functions, after which the core pointproton radius and corrections of Eq. (3) are applied to obtain the absolute charge radius. We note that induced two-body corrections to the radius operator are included naturally in the VS-IM-SRG formalism.

\section{A. Ground-state energies and radii}

Given the remarkable agreement with experimental ground-state energies for closed-shell systems from the

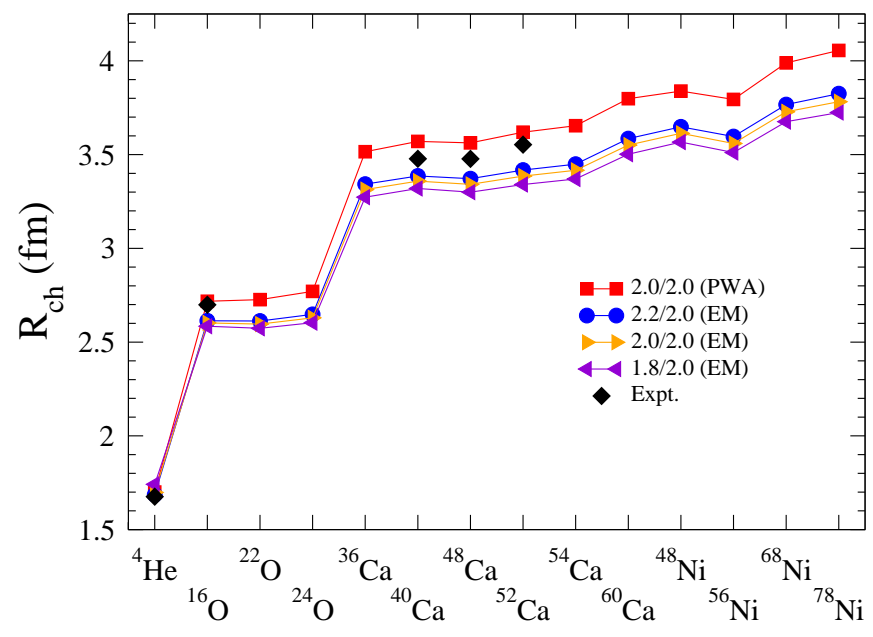

FIG. 6. Systematics of charge radii of closed-shell nuclei from ${ }^{4} \mathrm{He}$ to ${ }^{78} \mathrm{Ni}$ calculated with the IM-SRG for the four Hamiltonians considered. The results are compared against experimental charge radii [4] where available.

1.8/2.0 (EM) interaction discussed in Sec. II] we compare the systematics of ground-state energies calculated with this interaction to experimental data where they exist in the isotopic chains mentioned above. For sodium, sulfur, and calcium we take $e_{\operatorname{Max}} / E_{3 \operatorname{Max}}=12 / 16$, while for manganese and nickel we use $e_{\operatorname{Max}} / E_{3 \mathrm{Max}}=14 / 16$. In all cases $\hbar \Omega=16 \mathrm{MeV}$ is taken for the harmonicoscillator frequency. In addition, we directly compare single-reference IM-SRG and valence-space results in calcium and nickel for the closed-shell cases. The valence space is defined to be one major harmonic-oscillator shell for protons and neutrons. For example, for the sulfur chain we take a proton and neutron $s d$ valence space above an ${ }^{16} \mathrm{O}$ core for $N<20$, a proton $s d$ valence space above a ${ }^{28} \mathrm{O}$ core for $N=20$, and a proton $s d$ neutron $p f$ valence space above a ${ }^{28} \mathrm{O}$ core for $N>20$. It should be noted that at oscillator shell closures for neutrons, no explicit neutron excitations are allowed in the valence space. In particular for systems near the transition from one valence space to another, con- tributions from crossshell excitations will be important. These excitations are incorporated approximately by the IM-SRG decoupling, and our truncation to two-body operators is insufficient for these isotopes. We mark these oscillator closures as a vertical dotted line in all figures. While preliminary efforts indicate that the VS-IM-SRG approach is capable of decoupling the relevant mixed va- lence spaces, and thus treating these excitations explicitly, we will address this issue in a future work.

Beginning in the $s d$ region, we show in Figs. 7 and 8 , ground-state energies and two-neutron separation energies $S_{2 n}$ for sodium and sulfur isotopes, respectively. In both cases we find good agreement with absolute experimental ground-state energies, outside of ${ }^{31,32} \mathrm{Na}$, which are somewhat underbound. The ground states of ${ }^{30-32} \mathrm{Na}$ are dominated by deformed configurations [46, 47], not 

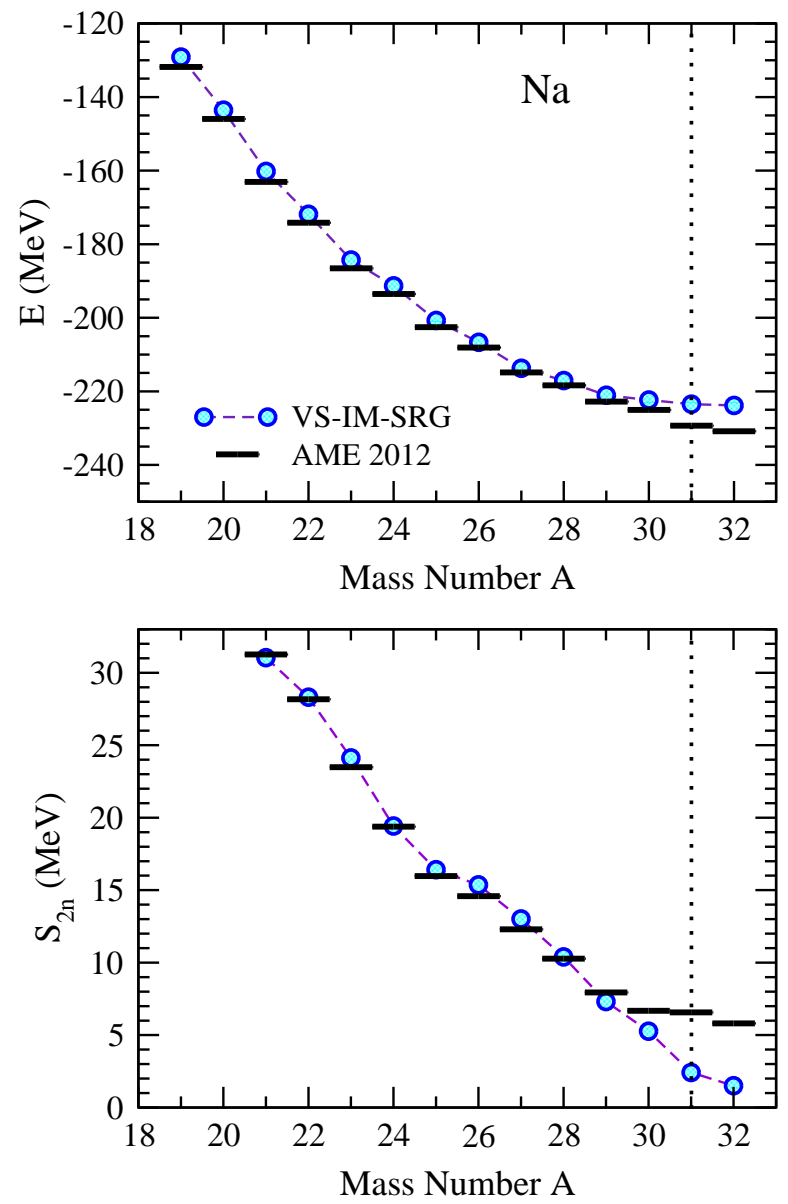

FIG. 7. Ground-state energies (top) and two-neutron separation energies (bottom) of sodium isotopes for the 1.8/2.0 (EM) Hamiltonian (circles) compared to experiment (AME 2012, bars) [40]. See text for details on the valence spaces used. The vertical dotted line marks the end of the $s d$ shell at $N=20$.

captured in neutron $s d$ or $p f$ valence-space calculations. These island-of-inversion isotopes will be investigated further in the context of decoupling neutron $s d-p f$ cross-shell valence spaces. Likewise, the $S_{2 n}$ results are in remarkable agreement with data, except in the region near $N=20$. In Ref. 12, sodium isotopes were also investigated with the VS-IM-SRG approach, but instead using the EM $500 \mathrm{MeV}$ potential with local $\mathrm{N}^{2} \mathrm{LO} 3 \mathrm{~N}$ forces [48] consistently SRG evolved to $\lambda=1.88 \mathrm{fm}^{-1}$. For this choice of Hamiltonian the isotopes ${ }^{22} \mathrm{Na}$ up to ${ }^{32} \mathrm{Na}$ are overbound, while the rest of the chain is in good agreement with experiment. With three protons above the closed $Z=8$ proton shell, no other ab initio method is currently able to calculate sodium isotopes. Except for the single-reference and VS-IM-SRG calculations of ${ }^{32,36} \mathrm{~S}$ with the SRG-evolved $\mathrm{NN}+3 \mathrm{~N}$ forces mentioned above [12], which display significant overbinding not seen with the 1.8/2.0 (EM) interaction used here, there are no other ab initio calculations available for these open-shell sulfur isotopes.
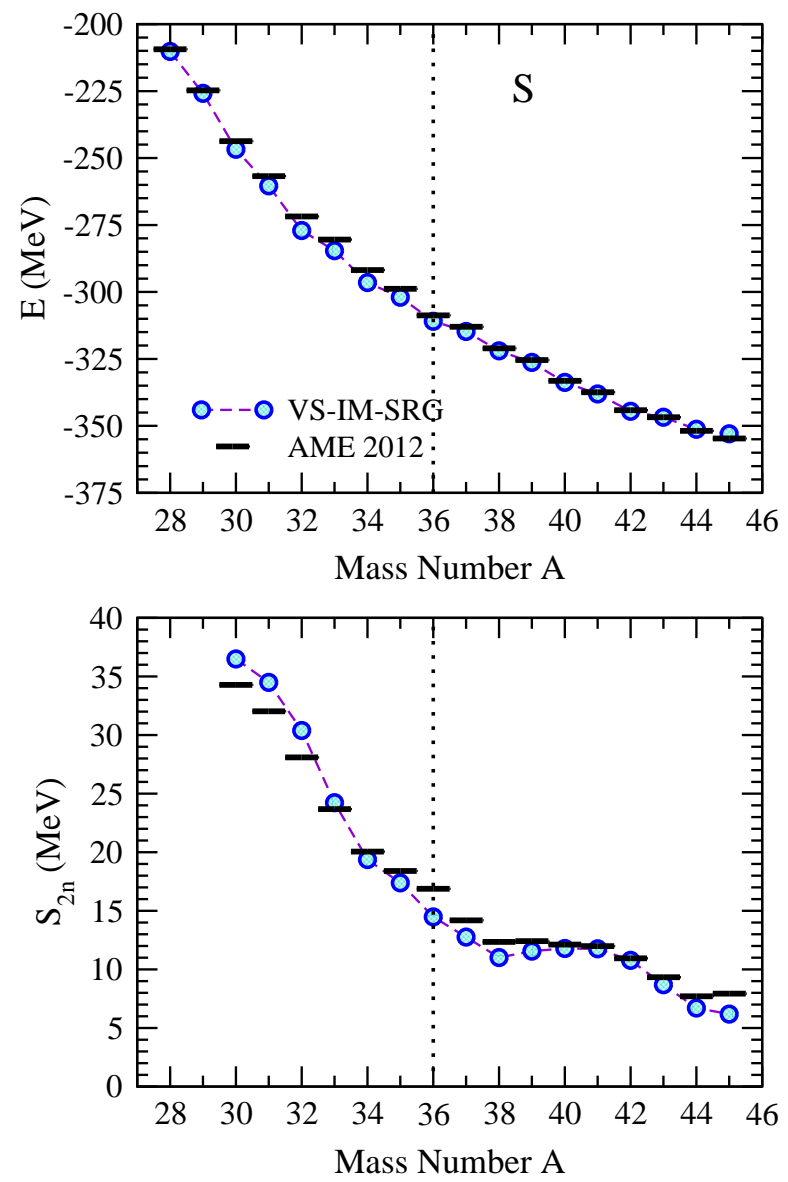

FIG. 8. Ground-state energies (top) and two-neutron separation energies (bottom) of sulfur isotopes compared to experiment, with the same legend and details as in Fig. 7.

In the $p f$ shell, the agreement with experimental data remains good as well, as shown in Figs. 9, 10, and 11 for calcium, manganese, and nickel isotopic chains, respectively. In calcium, we also compare with the corresponding single-reference results for ${ }^{40,48,52,54} \mathrm{Ca}$, where, as noted in Ref. [12], the VS-IM-SRG results agree with the single-reference calculations to better than $1 \%$. We also reproduce well the sharp decreases in $S_{2 n}$ values after $N=28$ and $N=32$, in good agreement with recent precision experiments [49, 50, indicating that the shell closures at $N=28$ and $N=32$ are well reproduced with the 1.8/2.0 (EM) interaction. We therefore also expect predictions of $S_{2 \mathrm{n}}$ values past $N=34$ to be reliable, at least qualitatively, when the data become available. Similar good agreement is seen for all trends in the manganese isotopes, which, with five protons above the $Z=20$ proton shell closure, are currently inaccessible to all other ab initio methods. Finally, we see that throughout the nickel chain, absolute ground-state energies become modestly overbound in the mid-shell region on the order of up to $10 \mathrm{MeV}$. All other experimental trends (aside from the artificial kink in the vicinity of $N=40$ ) are well reproduced, including the sharp drop 

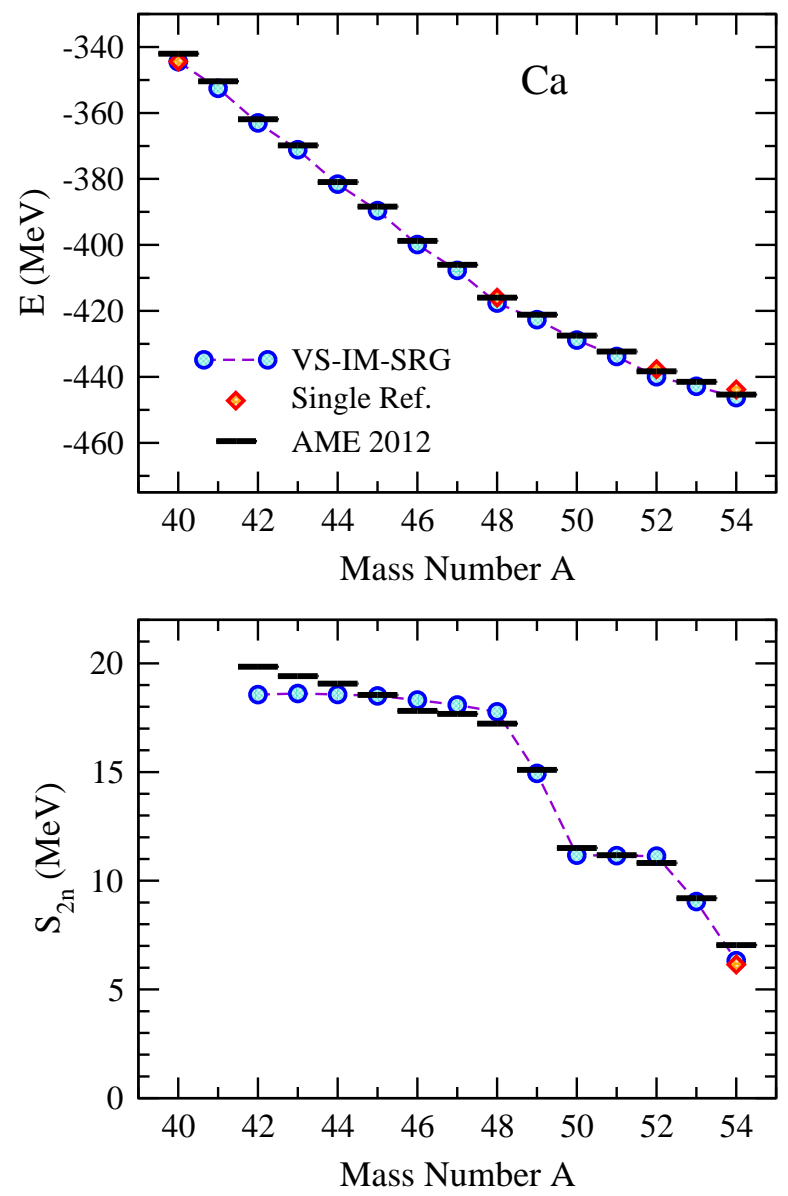

FIG. 9. Ground-state energies (top) and two-neutron separation energies (bottom) of calcium isotopes for the 1.8/2.0 (EM) Hamiltonian (circles) compared to experiment (AME 2012, bars) [40]. See text for details on the valence spaces used. For closed-subshell isotopes we also show the results of the single-reference IM-SRG (diamonds) for comparison.

past $N=28$. We also note that the somewhat larger discrepancy between single-reference and VS-IM-SRG results for ${ }^{56} \mathrm{Ni}$ is likely due to the ground-state configuration obtained in the valence-space diagonalization being only $30 \%$ pure filled proton and neutron $f_{7 / 2}$. While experimental energies are known past $A=72$, this represents our current limitation of diagonalizing the valencespace Hamiltonian exactly with modest computational resources. Using standard extensions and/or controlled truncations, isotopes as heavy as ${ }^{80} \mathrm{Ni}$ may be reached, though as seen in Sec. III such results may not be completely converged in terms of $E_{3 \mathrm{Max}}$. The results for the calcium and nickel isotopes using the consistently SRG-evolved EM $500 \mathrm{MeV}$ potential with local $\mathrm{N}^{2} \mathrm{LO} 3 \mathrm{~N}$ forces [48] are significantly overbound up to $100 \mathrm{MeV}$ [12, highlighting the importance of considering saturation for whether chiral interactions can describe bulk properties of nuclei across the nuclear chart.

While we reserve a complete discussion of charge radii systematics within the VS-IM-SRG for a future work, we
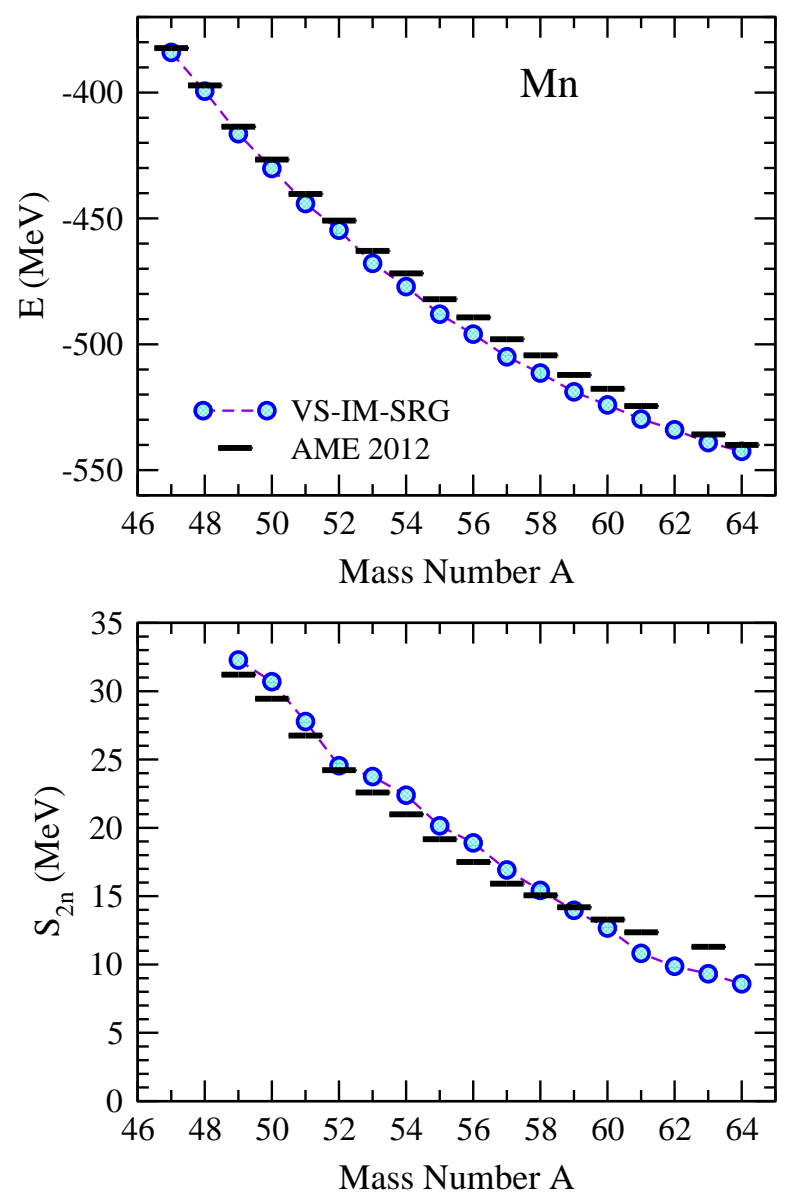

FIG. 10. Ground-state energies (top) and two-neutron separation energies (bottom) of manganese isotopes compared to experiment, with the same legend and details as in Fig. 9

illustrate the versatility of this approach in Fig. 12, where we plot radii for the complete $p f$-shell manganese chain, comparing with experimental data where available. From Fig. 6, we expect charge radii predicted with the 1.8/2.0 (EM) and 2.0/2.0 (PWA) interactions to be systematically too small and too large, respectively. While this is indeed seen, an interesting trend in charge radii is predicted in both cases, with a roughly parabolic shape to $N=28$, followed by a sharp increase for $N>28$. Experimental data are quite limited in manganese isotopes, but available data do seem to show this trend, albeit with more pronounced structures, as also seen in recent experimental measurements of charge radii in calcium isotopes [35. While neither interaction perfectly reproduces experiment, 2.0/2.0 (PWA) only moderately overpredicts charge radii and should provide a reasonably reliable guide to trends across isotopic chains. The general absence of systematic data highlights the importance of continued systematic experimental investigations of charge radii. 

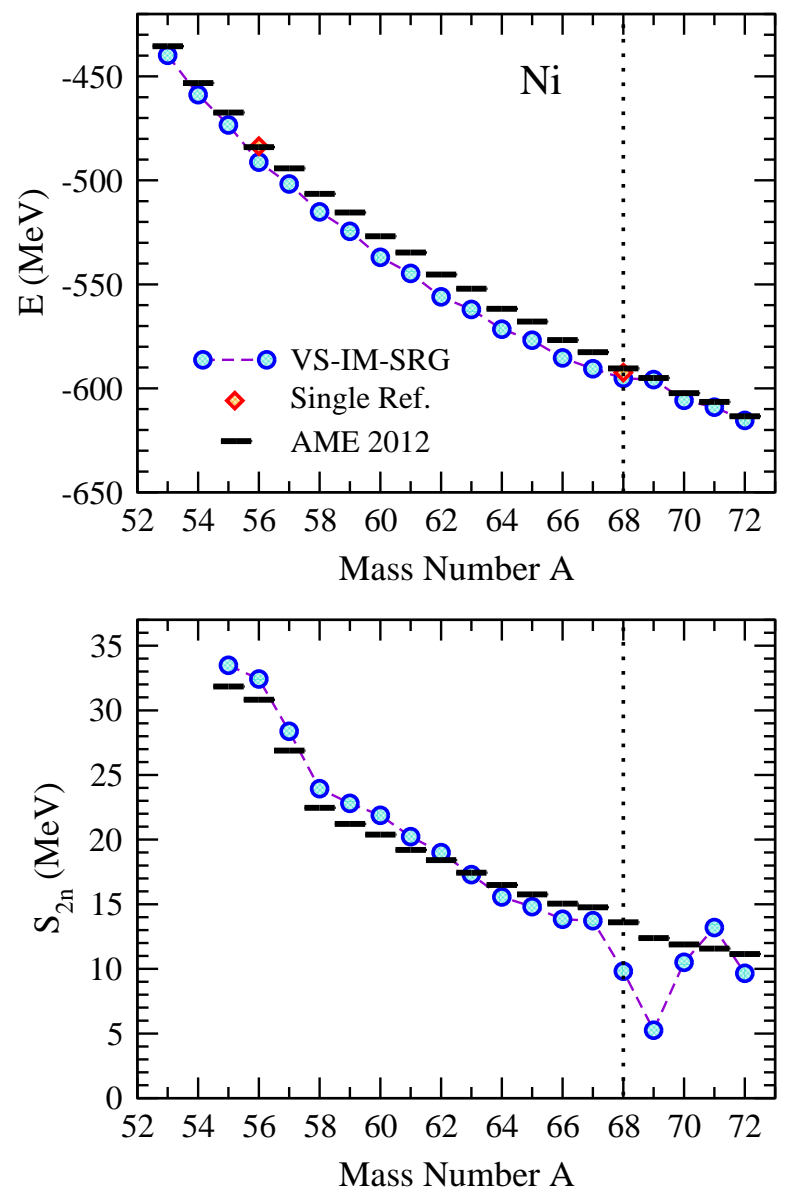

FIG. 11. Ground-state energies (top) and two-neutron separation energies (bottom) of nickel isotopes compared to experiment, with the same legend and details as in Fig. 9. The vertical dotted line marks the end of the $p f$ shell at $N=40$.

\section{B. Excited states}

Given the remarkable description of experimental ground-state properties from the 1.8/2.0 (EM) interaction, it is also of interest to investigate to what extent the structure of excited states is captured. In the VS-IMSRG approach, all excited states allowed within a given valence space are obtained directly via diagonalization. Here we focus on first excited $2^{+}$states and associated shell closures in the subset of even-even sulfur, calcium, and nickel isotopes.

Beginning with sulfur, shown in Fig. 13 , we see an overall good reproduction of the experimental trends in $2^{+}$ energies. When neutrons occupy the $s d$ valence space, however, these energies are systematically several hundred keV too high. Beyond $N=20$, when the neutron valence space changes to the $p f$ shell, agreement with data improves, including the modest peak at $N=28$ in ${ }^{44} \mathrm{~S}$. Given the absence of allowed neutron excitations at $N=20$, the $2^{+}$energy here is artificially too high and is expected to decrease when such degrees of freedom are

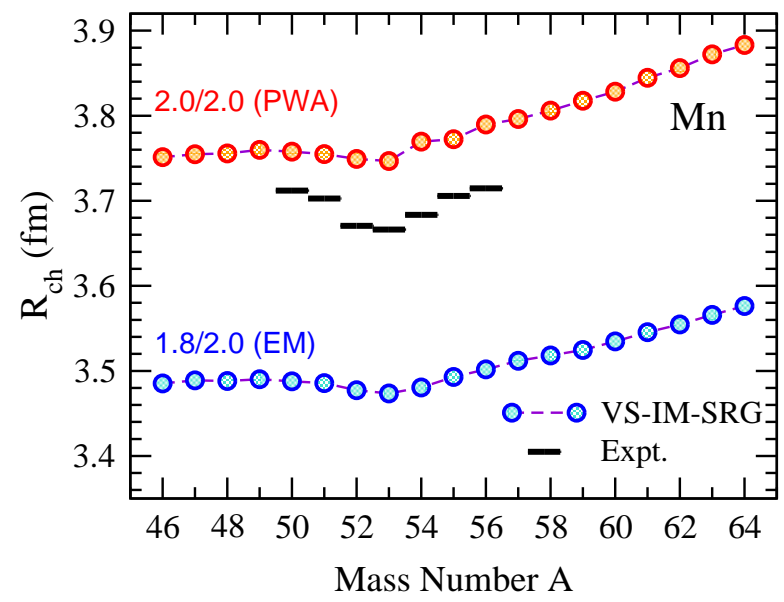

FIG. 12. Charge radii of manganese isotopes for the $1.8 / 2.0$ (EM) (blue circles) and 2.0/2.0 (PWA) (red circles) Hamiltonian compared to experiment 44]. See text for details on the valence spaces used.

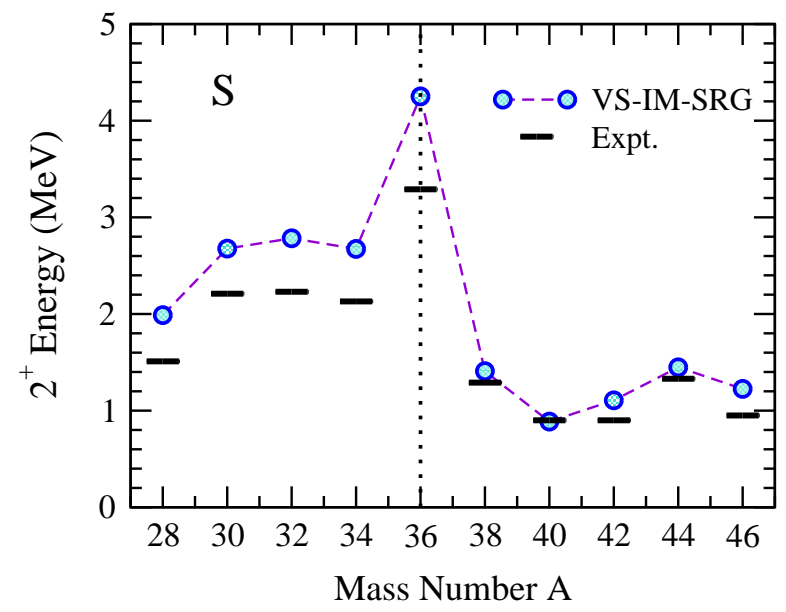

FIG. 13. First excited $2^{+}$energies of even sulfur isotopes for the 1.8/2.0 (EM) Hamiltonian (circles) compared to experiment [51. See text for details on the valence spaces used. The vertical dotted line marks the end of the $s d$ shell at $N=20$.

included in the valence space.

For the calcium isotopes, shown in Fig. 14, the calculated results agree well with data for open-shell cases. While relative peaks are seen at the $N=28,32$ shell closures as well as the recently measured $N=34$ closure in ${ }^{54} \mathrm{Ca}$ 52, they are systematically too high, particularly in ${ }^{48} \mathrm{Ca}$. While we might initially attribute this to neglected proton excitations due to the choice of valence space, similar features are also seen in the nickel isotopes, which allow both proton and neutron excitations except at $N=40$. For the nickel isotopes, a similar picture to sulfur is seen in Fig. 15 . Where when neutrons fill the $p f$ shell, the $2^{+}$energies reproduce the experimental trend, but are systematically several hundred $\mathrm{keV}$ too high. When neutrons begin filling the $s d g$ orbits past ${ }^{68} \mathrm{Ni}$, the results agree very well with data, making pre- 


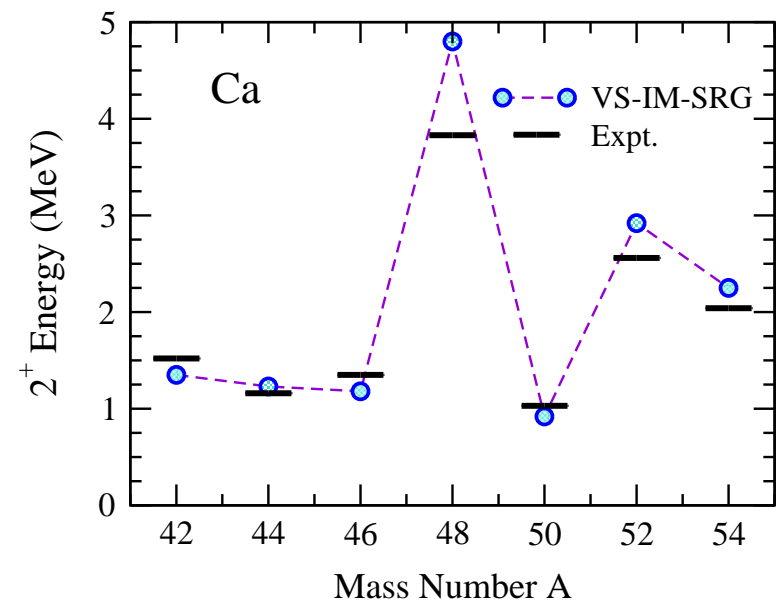

FIG. 14. First excited $2^{+}$energies of even calcium isotopes for the 1.8/2.0 (EM) Hamiltonian (circles) compared to experiment [51]. See text for details on the valence spaces used.

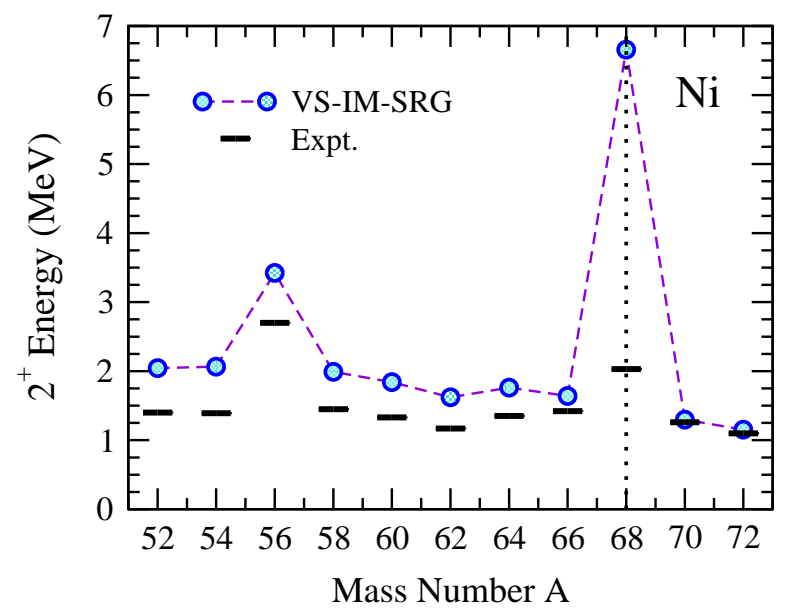

FIG. 15. First excited $2^{+}$energies of even nickel isotopes for the 1.8/2.0 (EM) Hamiltonian (circles) compared to experiment [51]. See text for details on the valence spaces used. The vertical dotted line marks the end of the $p f$ shell at $N=40$.

dictions out to ${ }^{80} \mathrm{Ni}$ possible to investigate the closedshell nature of ${ }^{78} \mathrm{Ni}$. The very high $2^{+}$state in ${ }^{68} \mathrm{Ni}$ is clearly due to a lack of allowed neutron excitations, and should again be as an artifact of the many-body approximation.

The pattern of too-high $2^{+}$energies in closed-shell systems is a common feature of our calculations. The origin of this behavior is unclear, but some direction might be provided by coupled-cluster calculations of ${ }^{48} \mathrm{Ca}$ and ${ }^{78} \mathrm{Ni}[36$. In this work, it was found that using the same 1.8/2.0 (EM) interaction, when particle-hole excitations were limited to the coupled-cluster singles and doubles (CCSD) approximation, the first excited $2^{+}$state in ${ }^{48} \mathrm{Ca}$ was approximately $1 \mathrm{MeV}$ too high, very close to our result. When perturbative triples excitations were then included, this energy was lowered to close to the experi- mental value, and a similar decrease was seen in the first $2^{+}$energy in ${ }^{78} \mathrm{Ni}$. Because the IM-SRG(2) approximation in this work is analogous to the CCSD truncation [7, we might expect a similar improvement in extensions of IM-SRG(2) analogous to the perturbative triples of coupled cluster. Due to the final step of diagonalizing in the valence space, however, the expectation would be that much of this physics should already be captured, as long as excitations near the Fermi surface were of dominant importance. As the development of a controlled approximation to IM-SRG(3) is currently in progress, we so far have no means to check whether such an improvement will remedy the too-high $2^{+}$states at closed shells. Nevertheless, this appears to be a deficiency in the manybody method, not the interaction.

\section{SUMMARY AND CONCLUSIONS}

We have performed ab initio IM-SRG calculations of ground-state energies and charge radii of broad range of closed- and open-shell nuclei with $A \leqslant 78$. For radii of open-shell nuclei, these represent first valence-space IM-SRG results. We have focused on a set of chiral lowresolution $\mathrm{NN}+3 \mathrm{~N}$ interactions that predict realistic saturation properties. As a baseline, we have first studied the convergence properties of these Hamiltonians with respect to model-space truncations for both two- and threebody interactions. Each of the NN+3N interactions used here reproduces few-body data with equivalent accuracy. However, the interactions do not produce equivalent results for medium-mass nuclei. In fact, the systematics of ground-state energies and radii indicates that the difference is dominantly due to their different nuclear matter saturation properties.

One particular interaction yields energies in good agreement with experiment from light nuclei up to the point at which we are limited by the convergence of the many-body calculation. This behavior appears to be accidental, in the sense that we could not have anticipated from the few-body results which of these interactions would produce the desired absolute energies, but it suggests two conclusions. First, that the operator structures contained in these chiral interactions $\left(\mathrm{NN}\right.$ at $\mathrm{N}^{3} \mathrm{LO}$ and $3 \mathrm{~N}$ at $\mathrm{N}^{2} \mathrm{LO}$ ) are sufficient to describe many of the features of the energies of light and medium-mass nuclei, while future consistent calculations at $\mathrm{N}^{3} \mathrm{LO}$ (and $\mathrm{N}^{2} \mathrm{LO}$ ) are of course called for. Second, as suggested in Ref. [25, saturation properties are essential for this accurate description. Both of these points highlight the importance of nuclear matter as a theoretical benchmark and guidance for the development of next-generation chiral interactions. 


\section{ACKNOWLEDGMENTS}

This work was supported by the ERC Grant No. 307986 STRONGINT, the DFG under Grant SFB 1245, the BMBF under Contract No. 05P15RDFN1, the National Research Council of Canada and NSERC. Com- putations were performed with an allocation of computing resources at the Jülich Supercomputing Center (JURECA), at the Computing Center of the TU Darmstadt (Lichtenberg), and at the Max-Planck-Institute for $\mathrm{Nu}-$ clear Physics.
[1] E. Epelbaum, H.-W. Hammer, and U.-G. Meißner, Rev. Mod. Phys. 81, 1773 (2009).

[2] R. Machleidt and D. R. Entem, Phys. Rep. 503, 1 (2011).

[3] S. K. Bogner, R. J. Furnstahl, and A. Schwenk, Prog. Part. Nucl. Phys. 65, 94 (2010).

[4] R. J. Furnstahl and K. Hebeler, Rep. Prog. Phys. 76, 126301 (2013).

[5] G. Hagen, T. Papenbrock, M. Hjorth-Jensen, and D. J. Dean, Rep. Prog. Phys. 77, 096302 (2014).

[6] V. Somà, A. Cipollone, C. Barbieri, P. Navrátil, and T. Duguet, Phys. Rev. C 89, 061301(R) (2014).

[7] H. Hergert, S. K. Bogner, T. D. Morris, A. Schwenk, and K. Tsukiyama, Phys. Rep. 621, 165 (2016).

[8] S. K. Bogner, H. Hergert, J. D. Holt, A. Schwenk, S. Binder, A. Calci, J. Langhammer, and R. Roth, Phys. Rev. Lett. 113, 142501 (2014).

[9] G. R. Jansen, J. Engel, G. Hagen, P. Navrátil, and A. Signoracci, Phys. Rev. Lett. 113, 142502 (2014).

[10] G. R. Jansen, A. Signoracci, G. Hagen, and P. Navrátil, Phys. Rev. C 94, 011301(R) (2016).

[11] S. R. Stroberg, H. Hergert, J. D. Holt, S. K. Bogner, and A. Schwenk, Phys. Rev. C 93, 051301(R) (2016).

[12] S. R. Stroberg, A. Calci, H. Hergert, J. D. Holt, S. K. Bogner, R. Roth, and A. Schwenk, Phys. Rev. Lett. 118, 032502 (2017).

[13] H.-W. Hammer, A. Nogga, and A. Schwenk, Rev. Mod. Phys. 85, 197 (2013).

[14] K. Hebeler, J. D. Holt, J. Menéndez, and A. Schwenk, Ann. Rev. Nucl. Part. Sci. 65, 457 (2015).

[15] S. K. Bogner, A. Schwenk, R. J. Furnstahl, and A. Nogga, Nucl. Phys. A 763, 59 (2005).

[16] K. Hebeler, S. K. Bogner, R. J. Furnstahl, A. Nogga, and A. Schwenk, Phys. Rev. C 83, 031301(R) (2011).

[17] G. Hagen, T. Papenbrock, A. Ekström, K. Wendt, G. Baardsen, S. Gandolfi, M. Hjorth-Jensen, and C. J. Horowitz, Phys. Rev. C 89, 014319 (2014).

[18] A. Carbone, A. Rios, and A. Polls, Phys. Rev. C 88, 044302 (2013).

[19] L. Coraggio, J. W. Holt, N. Itaco, R. Machleidt, L. E. Marcucci, and F. Sammarruca, Phys. Rev. C 89, 044321 (2014).

[20] J. A. Wheeler, Phys. Rev. 52, 1083 (1937).

[21] S. D. Drell and K. Huang, Phys. Rev. 91, 1527 (1953).

[22] R. Roth, A. Calci, J. Langhammer, and S. Binder, Phys. Rev. C 90, 024325 (2014).

[23] A. Cipollone, C. Barbieri, and P. Navrátil, Phys. Rev. Lett. 111, 062501 (2013).

[24] S. Binder, J. Langhammer, A. Calci, and R. Roth, Phys. Lett. B 736, 119 (2014).

[25] A. Ekström, G. R. Jansen, K. A. Wendt, G. Hagen,
T. Papenbrock, B. D. Carlsson, C. Forssén, M. HjorthJensen, P. Navrátil, and W. Nazarewicz, Phys. Rev. C 91, 051301(R) (2015).

[26] D. R. Entem and R. Machleidt, Phys. Rev. C 68, 041001(R) (2003)

[27] S. K. Bogner, R. J. Furnstahl, and R. J. Perry, Phys. Rev. C 75, 061001(R) (2007).

[28] U. van Kolck, Phys. Rev. C 49, 2932 (1994).

[29] E. Epelbaum, A. Nogga, W. Glöckle, H. Kamada, U.G. Meißner, and H. Witała, Phys. Rev. C 66, 064001 (2002).

[30] M. C. M. Rentmeester, R. G. E. Timmermans, and J. J. de Swart, Phys. Rev. C 67, 044001 (2003).

[31] C. Drischler, V. Somà, and A. Schwenk, Phys. Rev. C 89, 025806 (2014).

[32] C. Drischler, K. Hebeler, and A. Schwenk, Phys. Rev. C 93, 054314 (2016).

[33] J. Simonis, K. Hebeler, J. D. Holt, J. Menéndez, and A. Schwenk, Phys. Rev. C 93, 011302(R) (2016).

[34] G. Hagen et al., Nature Phys. 12, 186 (2016).

[35] R. F. Garcia Ruiz et al., Nature Phys. 12, 594 (2016).

[36] G. Hagen, G. R. Jansen, and T. Papenbrock, Phys. Rev. Lett. 117, 172501 (2016).

[37] T. D. Morris, N. Parzuchowski, and S. K. Bogner, Phys. Rev. C 92, 034331 (2015).

[38] K. Tsukiyama, S. K. Bogner, and A. Schwenk, Phys. Rev. C 85, 061304(R) (2012).

[39] B. Brown and W. Rae, Nuclear Data Sheets 120, 115 (2014).

[40] M. Wang, G. Audi, A. H. Wapstra, F. G. Kondev, M. MacCormick, X. Xu, and B. Pfeiffer, Chin. Phys. C 36, 1603 (2012).

[41] G. P. Kamuntavicius, Phys. Rev. C 56, 191 (1997).

[42] C. Patrignani et al. (Particle Data Group), Chin. Phys. C 40, 100001 (2016).

[43] A. Ong, J. C. Berengut, and V. V. Flambaum, Phys. Rev. C 82, 014320 (2010).

[44] I. Angeli and K. Marinova, Atomic Data and Nuclear Data Tables 99, 69 (2013).

[45] V. Lapoux, V. Somà, C. Barbieri, H. Hergert, J. D. Holt, and S. Stroberg, Phys. Rev. Lett. 117, 052501 (2016).

[46] V. Tripathi et al., Phys. Rev. C 76, 021301(R) (2007).

[47] P. Doornenbal et al., Phys. Rev. C 81, 041305(R) (2010).

[48] P. Navrátil, Few Body Syst. 41, 117 (2007).

[49] A. T. Gallant et al., Phys. Rev. Lett. 109, 032506 (2012).

[50] F. Wienholtz et al., Nature 498, 346 (2013).

[51] http://www.nndc.bnl.gov/ensdf/,.

[52] D. Steppenbeck et al., Nature 502, 207 (2013). 